Early dissociation of numbers and letters in the human brain

Sara Aurtenetxe1, Nicola Molinaro1,2, Doug Davidson1 \& Manuel Carreiras1,2,3

1. BCBL, Basque Center on Cognition, Brain and Language,

Donostia - San Sebastián, 2009, Spain

2. Ikerbasque, Basque foundation for science, Bilbao, Spain

3. Departamento de Lengua Vasca y Comunicación, Euskal Herriko

Unibertsitatea/Universidad del País Vasco, 48940 Leioa, Bizkaia, Spain 


\title{
Early dissociation of numbers and letters in the human brain
}

\section{Sara Aurtenetxe1, Nicola Molinaro1,2, Doug Davidson1 \& Manuel Carreiras1,2,3}

1. BCBL, Basque Center on Cognition, Brain and Language, Donostia - San Sebastián, 2009, Spain

2. Ikerbasque, Basque foundation for science, Bilbao, Spain

3. Departamento de Lengua Vasca y Comunicación, Euskal Herriko Unibertsitatea/Universidad del País Vasco, 48940 Leioa, Bizkaia, Spain

\begin{abstract}
Numbers and letters are culturally created symbols which are learned through repeated training. This experience leads to a functional specialization of the perceptual system of our brain. Recent evidence suggests a neural dissociation between these two symbols. While previous literature has shown that letters elicit a left lateralized neural response, new studies suggest that numbers elicit preferentially a bilateral or right lateralized response. However, the time course of the neural patterns that characterize this dissociation is still underspecified. In the present study, we investigated with magnetoencephalography (MEG) the spatio-temporal dynamics of the neural response generated by numbers, letters and perceptually matched false fonts presented visually. Twenty-five healthy adults were recorded while participants performed a dot detection task. By including two experiments, we were able to study the effects of single characters as well as those of strings of characters. The signal analysis was focused on the event related fields (ERF) of the MEG signal in the sensors and in the source space. The main results of our study showed an early ( $<200 \mathrm{~ms}$ ) preferential dissociation between single numbers and single letters on occipito-temporal sensors. When comparing strings of numbers and pseudowords, they differed also over prefrontal regions of the brain. These data offer a new example of acquired category-specific responses in the human brain.
\end{abstract}




\section{Introduction}

Numbers and letters are culturally created symbols that become meaningful only after extensive training -- they have no significance to infants or illiterate adults (Dehaene \& Cohen, 2007; Hamilton et al., 2006). The ability to recognize these constructs involves a functional preference of cognitive and perceptual systems and thus, offers a new example of acquired category-specific responses in the human brain.

Neural categorization of visual stimuli elicits a cascade of processes along the first hundreds of milliseconds after the presentation of the stimuli (Rossion et al., 2003; Tanaka et al., 1999). In the context of word forms, sublexical processing and orthographic-to-phonological conversion occur in early latencies after the stimulus onset (between 100-200 ms and 200-300 ms, respectively), whereas lexical access and semantic retrieval occur in later time-windows (between 300-400 ms and after $400 \mathrm{~ms}$ respectively) (Grainger and Holcomb 2009; Bann \& Herdman, 2016; Hauk et al., 2008). Specifically, visual encoding of letter/word forms occurs early around $130 \mathrm{~ms}$ from stimulus onset preferentially in the left ventral visual stream (McCandliss et al., 2003; Appelbaum, Liotti, Perez, Fox, \& Woldorff, 2009; Schendan, Ganis, \& Kutas, 1998). Differential anatomical and temporal patterns have been observed between single and strings of letters in later latencies (James et al., 2005; Park et al., 2012). However, further evidence at better temporal and anatomical resolution, and full-coverage methods would be valuable.

Recent evidence shows that the number-related pattern of neural activity dissociates from that of letters early during the first encoding levels in occipito-temporal regions of the brain (Park et al., 2014; Abboud et al., 2015; for a review see Hannagan et al. 2015). However, there is a lack of consistency regarding the hemispheric preference for such a dissociation. On the one side, opposite hemispheric recruitment of neurons has been evidenced for these two categories (Park et al., 2012). In an EEG study, Park et al., (2014) showed that while single numbers elicited increased EEG evoked responses compared to single letters on right hemispheric electrodes, the last evoked a similar pattern on left hemisphee electrodes. Both dissociations occurred in the time range of the N1 (between $140-170 \mathrm{~ms}$ ). By examining also the neural response to strings of characters, the authors showed that strings of letters elicited a left lateralized response around $250 \mathrm{~ms}$ (in the range of the P2) when comparing with strings of numbers. Similar hemispheric lateralized 
patterns for number and letter processing were observed in a source reconstruction in a magnetoencephalography (MEG) study performed by Carreiras et al., (2015). On the other hand, recent data shows that, instead of the unique right hemispheric specialization, numerals recruit populations of neurons in both hemispheres, bilaterally. In an fMRI study, Grotheer et al., (2016) observed a bilateral preferential response for numbers at the inferior temporal gyrus (ITG) when comparing with letters, false numbers or everyday objects. Similarly, Shum et al. (2013) observed with iEEG (intracranial electroencephalography) a region in the right that responded preferentially to numbers. Even though the coverage of the electrodes included mainly the right hemisphere, they found similar results in the left hemisphere as well.

Scientific work focused on the neural fingerprints of number processing has received less attention. Arabic digits have typically been used as control stimuli when studying letter- and word-specific neural activity. Also, to date, the handful of informative literature has included fMRI, EEG focusing on a limited number of electrodes, MEG restricting the analysis to regions of interest at the source space, and iEEG covering areas of the right hemisphere mainly. However, as far as we know, none of these studies have investigated the temporal dynamics and anatomical preferences of single and strings of numbers with a non-invasive technique which includes a high temporal and spatial resolution with MEG (by covering the whole head with 306 sensors ), and including sensor and source localization procedures and individual MRIs. Such an approach is important since the encoding and dissociation of visually presented stimuli potentially occurs on a millisecond scale and involves a variety of regions of the brain.

The purpose of the present study was to investigate the temporal dynamics and the anatomical localization of the dissociation of the neural evoked response to visually presented numbers, letters and false-fonts. To do so, we studied (in two experiments) the MEG signal in response to single numbers, letters and false fonts (Experiment 1), and strings of numbers, pseudowords and strings of false fonts (Experiment 2) in young adults during a dot-detection attention task. The task required participants to respond whenever a dot was presented (catch trials) among the stimuli. This low-level task was selected because it does not require explicit semantic or phonological processing but nevertheless ensures attention during the task. This way, participants can apply the same processing strategy for both numbers and letters, thus allowing direct comparison of these two conditions. This task has been used previously (Dehaene in Science 2010; Carreiras et al., 2015) 
so that it captures better the natural processing of the stimuli without requiring explicit additional processes such as lexical decision or semantic categorization.

In our analysis, first, we studied the stimulus evoked MEG signal in the sensor space. This analysis allowed us to estimate the time-window(s) where dissociations between the stimuli occur. Based on the previous evidence reported above and the data reported here, we suggest that numbers and letters can dissociate at early latencies during the first timewindows of the visual encoding ( $<200 \mathrm{~ms}$ after stimulus onset). We also show that single items and strings of stimuli can elicit differential neural patterns around 250-300 ms (Dehaene, 1995; Park et al., 2014). Second, we computed the source localization of these evoked responses and focused the analysis on the time-windows which were the most prominent in sensor space. This analysis allows us to estimate the source locations where the dissociation of numbers occurs.

\section{MATERIALS AND METHODS}

We report how we determined our sample size, all data exclusions, all inclusion/exclusion criteria, whether inclusion/exclusion criteria were established prior to data analysis, all manipulations, and all measures in the study.

Participants. 28 young adults were recruited for the present study (Park et al., 2014; Carreiras et al., 2015). From this initial sample, 3 participants were excluded due to a technical difficulty during acquisition. The final sample included a total of 25 participants (24+/-3 years of age). All of them reported to be native speakers of Spanish, right handed and free of neurological disease (criteria for participation established prior to data analysis). All participants gave their written informed consent in accordance with guidelines approved by the Research Committees of Basque Center on Cognition, Brain and Language. No part of the study procedures or analyses was pre-registered prior to the research being conducted.

\section{Experimental design}

Two experiments were administered in this study using Psychtoolbox. Both experiments included a visual detection tasks (Figure 1) and differed between them only in the stimuli. 


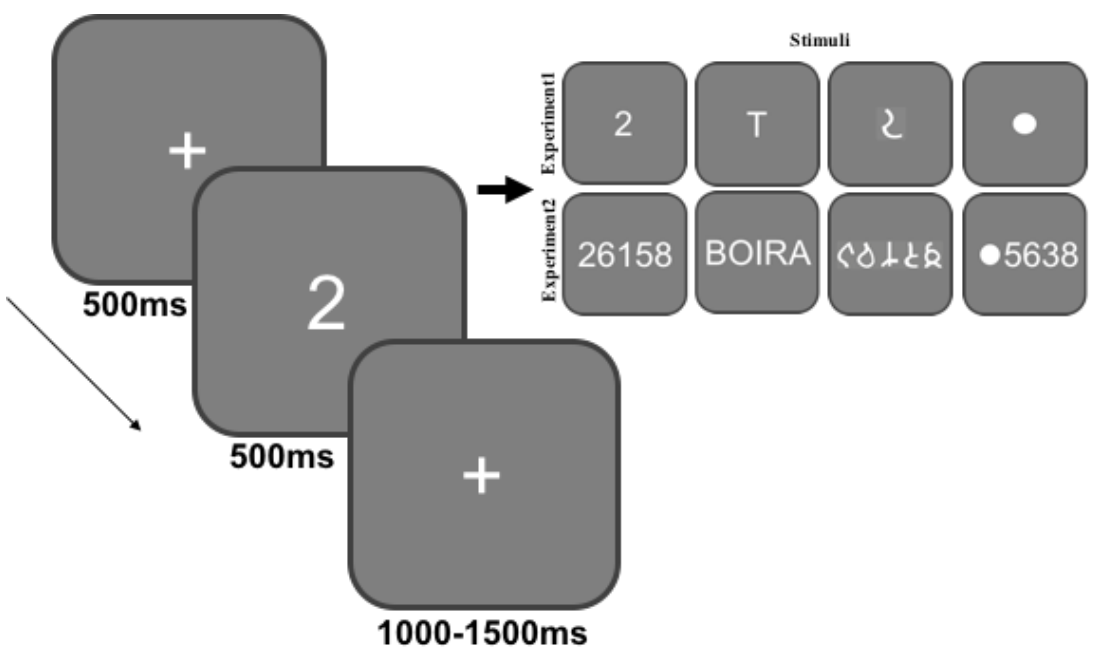

Figure 1. Visual detection task (left panel). Participants were instructed to attend to the stimuli and make a button press whenever a dot was presented. (b) Stimuli used in Experiment 1 (single numbers, single letters, single false-fonts and dot-detection) and in Experiment 2 (strings of numbers, pseudowords, strings of false-fonts and dot-detection).

Experiment 1. Participants were presented with three types of single stimuli (numbers, letters and false fonts). The numbers included digits from 1 to 9 , and the letters were A, C, D, F, L, P, S, U and V. Following the procedure by Shum et al., (2013), the false fonts were created from rearranged numeral and letter stimuli, with the number of pixels, angles, and curves kept as similar as possible while ensuring that the stimulus remained unrecognizable.

Experiment 2. The second experiment mimics the first with the difference that it included strings of stimuli (strings of numbers, pseudowords and strings of false fonts) instead of single-character stimuli. Each string included 5-6 letters, numbers, or false fonts. The strings of numbers consisted of combinations of digits between 1-9. Phonotactically legal pseudowords were used instead of consonant strings since the first could be clustered in a unified readable item, similar to what happens with number strings. The pseudowords were the following: ASIMA, BOIRA, DOBECA, DOCHAS, EGALO, MODRO, PLETAR, TEPOR, TOLAS.

For both experiments, the stimuli were presented in the center of the screen in a white font (Arial capital letters, covering not more that $1^{\circ}$ of visual angle on the screen positioned $\sim 1$ meter far from the participant) on a grey background. Each stimulus was repeated 22 times resulting in a total of 198 stimuli per condition. 
The trial started with a $500 \mathrm{~ms}$ baseline followed by the presentation of the stimuli for $500 \mathrm{~ms}$. After the stimulus offset, an intertrial interval varied between 1000-1500 ms and participants were invited to blink during this period. Participants were instructed to attend to the stimuli and report with a button press whenever a dot (catch trial) was presented. The catch trials were included in both experiments in order to ensure attention during the tasks. For Experiment 1, catch trials consisted of a sole dot, whereas for Experiment 2 the catch trials consisted of a string of numbers/letters/false fonts (depending on condition) and a dot (see Figure 1). During the entire experiment, participants were instructed to make a button press whenever a dot was present as a stimulus. This allowed us to ensure that participants were attending to the stimuli. The catch trials involved $10 \%$ of the total trials and were not included for the MEG signal analysis. Participants with accuracy lower than $80 \%$ in the catch trials would be excluded from the analysis.

The stimuli within each experiment were presented in random order. Participants were instructed to fixate on the center of the screen (fixation cross or stimulus) during the entire task.

\section{Data acquisition}

MEG data was continuously recorded (1000 Hz sample rate, $0.01-330 \mathrm{~Hz}$ online filter) during the performance of the attentional task using a 306-channel (102 magnetometers and 204 planar gradiometers) system (ElektaC, VectorView) placed in a magnetically shielded room (Vacuumschmelze GmbH, Hanau, Germany) at the Basque Center on Cognition, Brain and Language (Donostia-San Sebastián, Spain).

Individual head shapes were obtained by using a three-dimensional Fastrak digitizer (Polhemus). In addition, four head position indication (HPI) coils were placed in each subjects' head: two in the mastoids and two on the forehead. The HPI coils provided continuous head position estimation during the recording.

For source reconstruction, a high-resolution 3D structural MR image (T1-weighted MPRAGE sequence) was acquired with a 3T Trio MRI scanner (Siemens, Munich, Germany) to the individual participants. Due to technical reasons, we obtained MRI scans of 20 participants. For the remaining 5 participants, we used the MNI template MRI provided by Fieldtrip (see reference below). 


\section{Data analysis}

Both experiments were analyzed similarly. Maxfilter software (version 2.2., Elekta Neuromag) was used offline to reduce external noise and compensate for head movements (temporal extension of the signal space separation method; Taulu and Kajola, 2005; Taulu and Simola, 2006).

The data analysis of the MEG signal was performed using the Fieldtrip Matlab toolbox for $\quad$ analysis (Oostenveld et al., 2011; http://www.ru.nl/neuroimaging/fieldtrip). The analysis was conducted on the gradiometers. Only epochs free of button responses were included in the analysis. Automatic artifact rejection was applied to remove trials containing SQUID jumps and muscle activity. Independent component analysis (ICA) was used to visually detect and discard eye blinks and electrocardiogram activity from the MEG signal ("runica" algorithm implemented in FieldTrip/EEGLAB). The ICA procedure applies a linear decomposition to the data after which the data is represented as components. First, these components are visually inspected in a trial-by-trial basis and then, the trials affected by the artifacts are identified and rejected from the data. This procedure excludes the bad components and projects back the signal free of artifacts. The data was band-pass filtered between 1 and $35 \mathrm{~Hz}$, demeaned, detrended and segmented between $300 \mathrm{~ms}$ before and $500 \mathrm{~ms}$ after the stimulus onset (resulting in epochs of $800 \mathrm{~ms}$ ).

Sensor level analysis. The artifact-free signal was baseline corrected (with a $200 \mathrm{~ms}$ timewindow prior to stimulus onset) and averaged across trials resulting in an event related (ERF) for each sensor, condition and participant.

Statistical analysis. Paired-sample t-tests (two-tailed) were used to test the null hypothesis of no difference between the different conditions (numbers vs. letters, numbers vs. false fonts and letters vs. false fonts) in time and sensor locations. In order to control for the family-wise error rate in the context of multiple comparisons over time points and sensors, a cluster-based nonparametric permutation statistic was performed (Maris \& Oostenveld, 2007). Accordingly, clusters of channels and time samples with significant differences $(\mathrm{p}<0.025)$ were created by temporal and spatial adjacency. A set of 1000 permutations was created by randomly assigning condition labels and then $\mathrm{t}$ values were computed for each permutation. A cluster was considered to have a statistically significant effect if the sum of t-values in the original dataset was greater than 
the 95th percentile $(\mathrm{p}<0.05)$ of the distribution of the corresponding values in the randomized data. This analysis allowed us to establish the time-window(s) of interest for a following post-hoc two-tail paired-sample t-test. The resulting significant timewindow(s) were selected for the analysis at the source space.

We would like to note that although our findings are based on relatively common analysis procedures, recent papers have suggested that the standard statistical practice cannot grant conclusions about the experimental question under debate (Button et al. 2013; Gelman \& Carlin, 2014; Greenland et al. 2016; Wasserstein \& Lazar, 2016; Benjamin et al. 2018; Lakens et al. 2018; Sassenhagen \& Draschkow, 2019). The present results should be taken heuristically and the results of our exploratory analyses might be useful for planning future pre-registered studies of the same phenomena. Interested readers are encouraged to examine the data provided with this manuscript.

Source level analysis. Source reconstruction analysis was performed in order to localize the source of origin of the effects observed at the sensor level. For that aim, first a singleshell head model was constructed from the anatomical MRI. A template grid with $3 \mathrm{~mm}^{\wedge} 3$ spacing was constructed using a MNI template brain. Then, single subject grids were produced by warping the individual anatomical scans to this template and applying the inverse warp to the template grid. This produced source- level data aligned across subjects in MNI space.

Source reconstruction of the MEG signal was performed with Linearly Constrained Minimum Variance Beamformer (Zhang and Liu, 2015) following a common filter approach. The spatial filter's coefficients were obtained from the average covariance matrix from trials belonging to the three conditions. The resulting spatial filter coefficients were then applied to each condition separately. This procedure results in a power estimate per source location, time, condition and participant.

Statistical analysis. The statistical analysis at the source space was focused on the timewindows and contrasts showing the significant effects at the sensor level. To quantify the differences in power between these significant contrasts, paired-sample t-tests (onetailed) were used $(\mathrm{p}<0.05)$. A cluster-based permutation approach was used in order to control for multiple comparisons over grid points (as explained above). The same caution about the statistical results applies for the source analysis as above. 
Effect size. Effect size (Cohen's $d$ ) was computed to test the magnitude of the effects between the conditions in the sensors and in the source space with the following formula:

$$
\mathrm{d}=(\mu 2-\mu 1) / \sigma
$$

where $\mu_{2}$ and $\mu_{1}$ are the condition means and $\sigma$ is the pooled standard deviation of the 2 conditions. The pooled standard deviation was calculated as follows:

$$
\sigma=\operatorname{sqrt}\left(\left(\operatorname{sd} 1^{\wedge} 2+\operatorname{sd} 2^{\wedge} 2\right) / 2\right)
$$

where $s d 1$ and $s d 2$ are the standard deviations of the means of each condition.

In order to offer a complete view of the data, we also depict the means (M), standard deviations (SD), effect sizes and correlation values of the three conditions (numbers, letters, false-fonts), that result in the significant topographies (sensors) and latencies (time-windows) after contrasting the conditions statistically. For example, the data "comparison 1: numbers $\left[\mathrm{M}=.13^{-11} ; \mathrm{SD}=.75^{-12}\right]$ versus letters $\left[\mathrm{M}=.09^{-11} ; \mathrm{SD}=.69^{-12}\right]$, time-window: 124-208 ms, effect size: $-.54, \mathrm{r}=.91$ ", is calculated by taking the rawprocessed data (the data before calculating the statistics) and selecting the specific sensors and time-windows which resulted statistically significant when applying the statistical procedure. This procedure offers an overview of the raw-processed data just at the significant sensors and time-windows.

\section{Results}

\section{Behavior}

Experiment1. Participants on average responded to the catch trials with a $94 \pm 10$ percent accuracy and with a $495 \pm 125 \mathrm{~ms}$ delay $(\mathrm{M} \pm \mathrm{SD})$.

Experiment2. Participants on average responded to the catch trials with a $97 \pm 3$ percent accuracy and responded with a $489 \pm 144 \mathrm{~ms}$ delay(M $\pm \mathrm{SD})$. 


\section{Magnetoencephalography}

Experiment 1. Sensor space

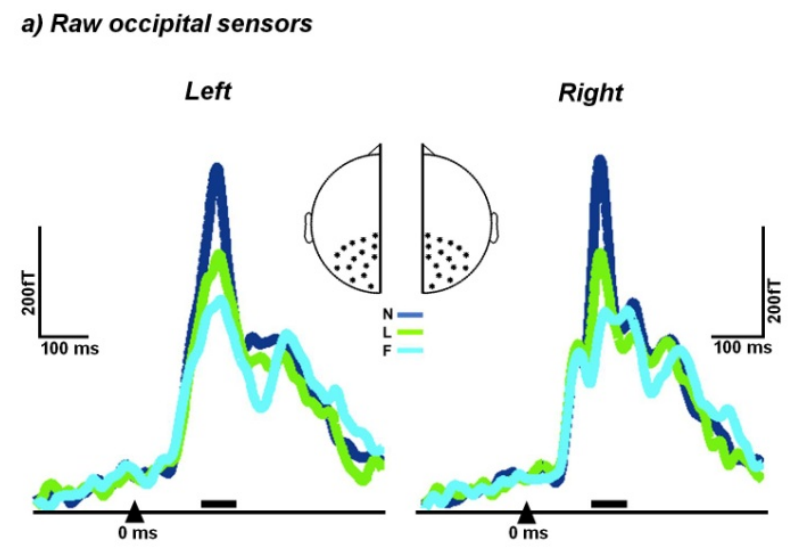

b) Statistics

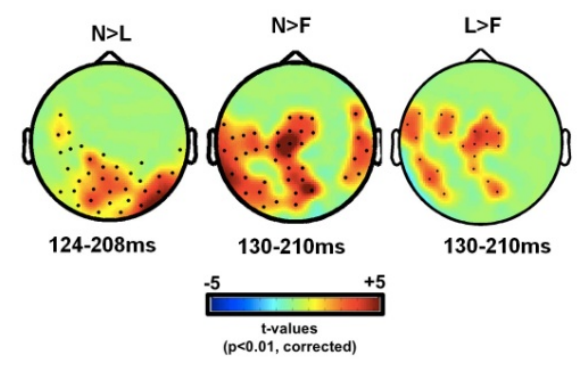

Figure2. Experiment1 sensors. a) Raw occipital sensors. Raw event related fields (ERF) averaged over the occipital sensors (left and right separately) for visualization purposes (dark blue: single numbers; green: single letters; light blue: single false fonts). The black triangle indicates the stimulus onset $(0 \mathrm{~ms})$. The black rectangle indicates the timing of the three significant time windows (124-208 ms, 130-210 ms and 130-210 ms) resulting from the $\mathrm{t}$ statistics between the conditions ( $\mathrm{p}<0.01$, corrected). b) Statistics. Topographies of the distribution of the significant t-statistics at the sensor level $(\mathrm{N}>\mathrm{L}$ : single numbers greater than single letters; $\mathrm{N}>\mathrm{F}$ : single numbers greater than single false fonts; $\mathrm{L}>\mathrm{F}$ : single letters greater than single false fonts). Dark dots display the significant sensors resulting from the paired-sample statistics. The colorbar displays the t-values.

In line with previous literature (Shum et al., 2013; Park et al., 2014), the present results show that the visual presentation of single numbers, letters and false fonts elicit a neural response which peaks around $160 \mathrm{~ms}$ after the stimulus onset on occipital and occipito-temporal sensors of the MEG. Numbers elicited the largest values in comparison with the rest of the stimuli. Results from the paired-sample t-tests revealed (Fig. 2) significant effects between the conditions over early time-windows ( $p<0.01$, corrected). When comparing with single letters, numbers elicited significant enhanced power over occipito-temporal sensors bilaterally (with higher effect over the right sensors) and over left temporal sensors (124-208 ms). Similarly, when comparing with single false fonts, numbers showed higher power values over left occipito-temporal sensors, over temporal 
sensors bilaterally and over central sensors (130-210 ms). When letters were compared with false fonts, the first elicited higher power values over left temporal and central sensors (130-210 ms).

The results of Experiment 1 in the sensors space (Figure 2) showed medium and large effects. Means (M), standard deviations (SD), effect sizes and correlation values of the three conditions (numbers, letters, false-fonts), that result in the significant topographies (sensors) and latencies (tw, time-windows) shown above, are depicted as follows: comparison 1) numbers $\left[\mathrm{M}=.13^{-11} ; \mathrm{SD}=.75^{-12}\right]$ versus letters $\left[\mathrm{M}=.09^{-11} ; \mathrm{SD}=.69^{-}\right.$ ${ }^{12}$ ], tw: 124-208 ms:, effect size: $-.54, \mathrm{r}=.91$; comparison 2) numbers $\left[\mathrm{M}=.98^{-12} ; \mathrm{SD}=.57^{-}\right.$ $\left.\left.{ }^{12}\right)\right]$ versus false fonts $\left[\mathrm{M}=.5^{-12} ; \mathrm{SD}=.42^{-12}\right]$, tw: $130-210 \mathrm{~ms}$, effect size: $-.95, \mathrm{r}=.84$; comparison 3) letters $\left[\mathrm{M}=.69^{-12} ; \mathrm{SD}=.47^{-12}\right]$ versus false fonts $\left[\mathrm{M}=.32^{-12} ; \mathrm{SD}=.4^{-12}\right]$, tw: 130-210 ms, effect size: $-.83, r=.86$.

\section{Experiment 1. Source space}

a) Number $>$ Letter (124-208ms)

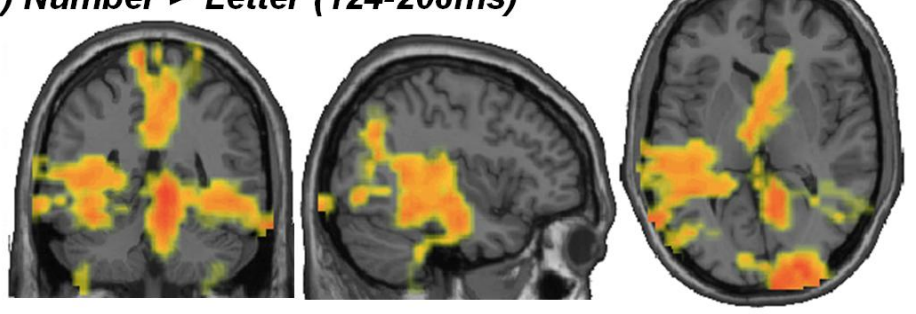

b) Number $>$ Falsefont (130-210ms)
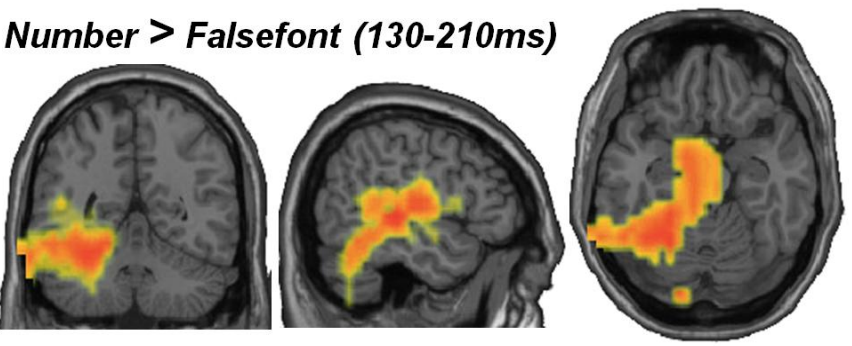

c) Letter $>$ Falsefont (130-210ms)
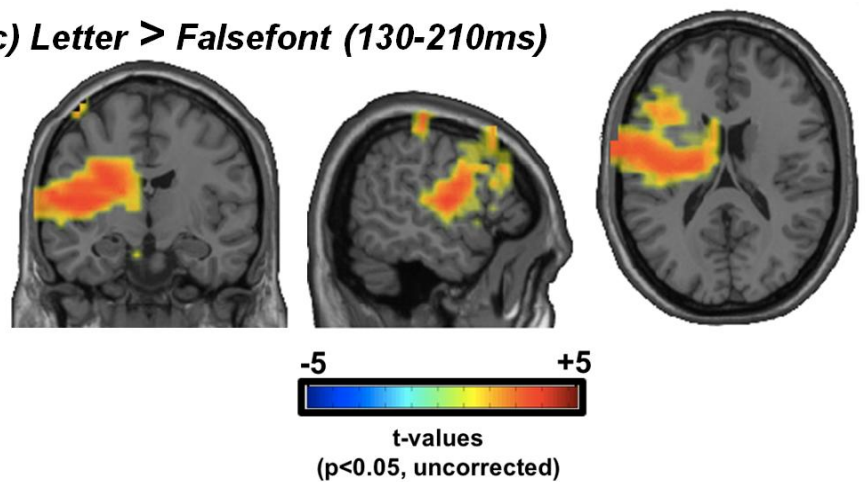
Figure3. Experiment1 sources. Cortical distribution of cluster-based statistical differences in brain activity between the conditions during the significant timewindows found at the sensor space. Note that only significant effects are shown.

\begin{tabular}{ll}
\hline Contrast & Coordinates $[\mathrm{x}, \mathrm{y}, \mathrm{z}]$ \\
\hline number $>$ letter & {$[-3.9,-4.7,0.3]$} \\
number $>$ falsefont & {$[-5,-5.3,-2]$} \\
letter $>$ falsefont & {$[-5.5,-1.1,1.7]$} \\
\hline
\end{tabular}

Table1. Coordinates of significant sources (MNI). The coordinates of the highest statistical values for each of the contrasts are shown.

Source space analysis was performed over the significant time-windows resulting from the sensor analysis. Paired-sample t-tests were computed between the conditions in order to test for significant differences between the conditions at the source space. In line with the results in sensor space, after selecting the a-priori time-windows of interest, the cluster-based permutation test revealed (Figure 3) a significant difference between the numbers and letters at the source space. These differences were most pronounced over the fusiform gyrus, middle temporal gyrus and auditory cortex of the left hemisphere, and over the inferior temporal gyrus and the secondary visual cortex in the right hemisphere. When testing for effects between numbers and false fonts, the cluster-based permutation test revealed a significant difference which was most pronounced over the fusiform and superior temporal gyrus of the left hemisphere. When testing for effects between letters with false fonts, the cluster-based permutation test revealed a significant difference which was most pronounced over the left inferior prefrontal cortex (and the premotor cortex).

The results of Experiment 1 in the source space (Figure 3) showed medium and large effects. Means (M), standard deviations (SD), effect sizes and correlation values of the three conditions (numbers, letters, false-fonts), that result in the significant sources at the significant time-windows (tw) in the sensor space are depicted as follows: comparison 1) numbers $[\mathrm{M}=2.94 ; \mathrm{SD} .8]$ versus letters $[\mathrm{M}=2.37 ; \mathrm{SD}=.6]$, tw: 124-208 ms, effect size: $-.8, \mathrm{r}=.84$; comparison 2 ) numbers $[\mathrm{M}=3.22 ; \mathrm{SD}=.8]$ versus false fonts $[\mathrm{M}=2.62 ; \mathrm{SD}=.8]$, tw: 130-210 ms, effect size: $-.74, \mathrm{r}=.35$; comparison 3) letters $[\mathrm{M}=2.26 ; \mathrm{SD}=.75]$ versus false fonts $[\mathrm{M}=1.6 ; \mathrm{SD}=.38]$, tw: 130-210 ms, effect size: $-1.09, \mathrm{r}=.61$. 


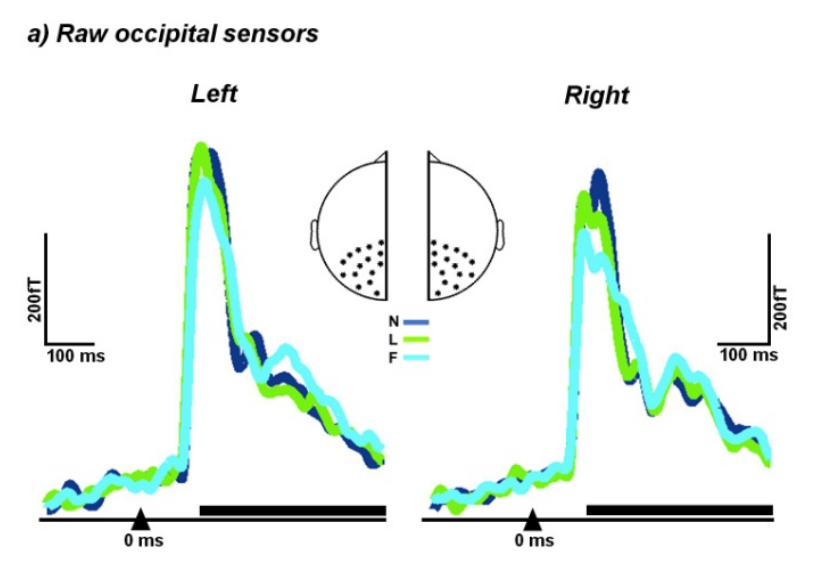

b) Statistics

Figure4. Experiment 2 sensors. a) Raw occipital sensors. Raw event related fields (ERF) averaged over the occipital sensors (left and right separately) for visualization purposes (dark blue: strings of numbers; green: pseudowords; light blue: strings of false fonts). The black triangle indicates the stimulus onset $(0 \mathrm{~ms})$. ). The black rectangle indicates the timing of the four significant time windows (264-500 ms, 93-191 ms, 180-276 ms, 171$262 \mathrm{~ms}$ ) resulting from the t-statistics between the conditions $(\mathrm{p}<0.01$, corrected). b) Statistics. Topographies of the distribution of the significant t-statistics at the sensor level $(\mathrm{N}<\mathrm{P}$ : strings of numbers lower than pseudowords; $\mathrm{N}>\mathrm{F}$ : strings of numbers greater than strings of false fonts; $\mathrm{N}<\mathrm{F}$ : strings of numbers lower than strings of false fonts; $\mathrm{P}>\mathrm{F}$ : pseudowords greater than strings of false fonts). Dark dots display the significant sensors resulting from the paired-sample statistics. The colorbar displays the t-values.

In line with a large previous literature (Park et al., 2012; 2014), the present results show that the visual presentation of number strings, letters strings and false font strings elicit a ERF response which peaks around $160 \mathrm{~ms}$ after the stimulus onset occipital and occipito-temporal sensors of the MEG. When testing for effects between the conditions, the paired-sample t-tests revealed (Fig. 3) significant effects between the conditions over early and late time-windows. Number strings elicited a smaller magnitude response than pseudowords during a late time-window $(264-500 \mathrm{~ms})$. The cluster was more pronounced over left temporal and frontal sensors. When testing for effects between numbers strings 
and false font strings, the cluster based permutation test revealed a positive and a negative cluster. During early time-windows (93-191 ms), number strings elicited enhanced power values than false font strings. The cluster was more pronounced over frontal, temporal and occipital sensors of both hemispheres. During later time-windows (180-276 ms), false font strings elicited a larger response than number strings. The cluster was more pronounced over temporal and occipital sensors of both hemispheres. The comparison between pseudowords and false font strings showed a stronger response for false fonts strings during later time-window (171-262 ms). The cluster was more pronounced over temporal and occipital sensors of the right hemisphere.

The results of Experiment 2 in the sensors space (Figure 4) showed medium and large effects. Means (M), standard deviations (SD), effect sizes and correlation values of the three conditions (numbers, letters, false-fonts), that result in the significant topographies (sensors) and latencies (tw, time-windows) shown above, are depicted as follows: comparison 1) numbers $\left[\mathrm{M}=.2^{-12} ; \mathrm{SD}=.34^{-12}\right]$ versus letters $\left[\mathrm{M}=.61^{-12} ; \mathrm{SD}=.49^{-}\right.$ ${ }^{12}$ ], tw: 264-500 ms, effect size: .96, $\mathrm{r}=.75$; comparison $2 \mathrm{~A}$ ) numbers $\left[\mathrm{M}=.015^{-12}\right.$; $\mathrm{SD}=.69^{-}$ $\left.{ }^{12}\right]$ versus false fonts $\left[\mathrm{M}=.011^{-12} ; \mathrm{SD}=.5^{-12}\right]$, tw: 93-191, effect size: $-.75, \mathrm{r}=87$; comparison $2 \mathrm{~B}$ ) numbers $\left[\mathrm{M}=.0006^{-12} ; \mathrm{SD}=.1^{-12}\right]$ versus false fonts $\left[\mathrm{M}=.011^{-12} ; \mathrm{SD}=.68^{-}\right.$ ${ }^{12}$, tw: 180-276, effect size: 2.26, $\mathrm{r}=.13$; comparison 3) letters $\left[\mathrm{M}=.48^{-12} ; \mathrm{SD}=.33^{-12}\right]$ versus false fonts $\left[\mathrm{M}=.98^{-12} ; \mathrm{SD}=.52^{-12}\right]$, tw: $171-262$, effect size: $1.14, \mathrm{r}=.79$. 
a) NumberString $<$ Pseudoword (264-500m
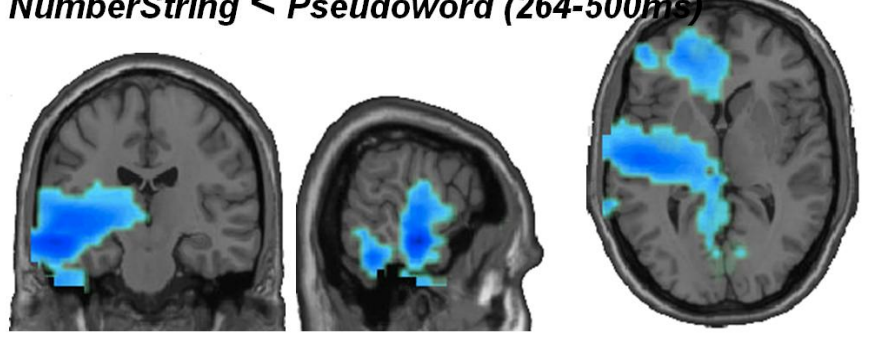

b) NumberString $>$ FalseFontString(93-191ps

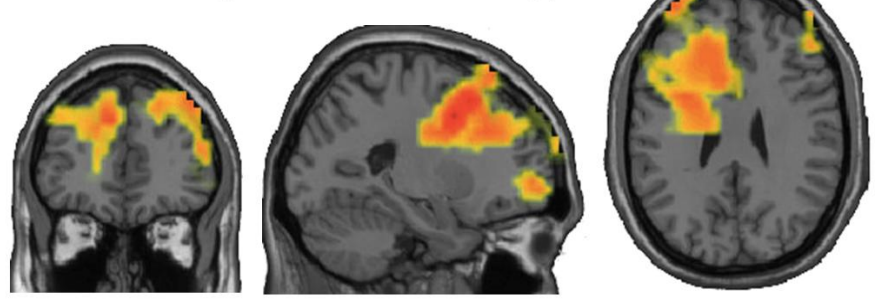

c) Pseudoword< FalseFontString (171-262
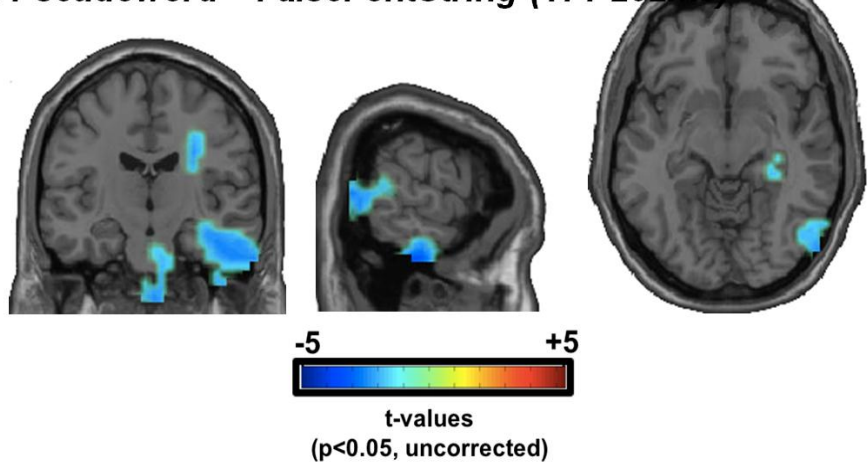

Figure5. Experiment 2 sources. Cortical distribution of cluster-based statistical differences in brain activity between the conditions during the significant timewindows found at the sensor space. Note that only significant effects are shown.

\begin{tabular}{ll}
\hline Contrast & Coordinates $[\mathbf{x}, \mathbf{y}, \mathbf{z}]$ \\
\hline numberstring $<$ pseudoword & {$[-6.1,-2,0.1]$} \\
numberstring $>$ falsefontstring & {$[-2.4,4.1,2.7]$} \\
pseudowrod $<$ falsefontstring & {$[6.5,-1.6,-1]$} \\
\hline
\end{tabular}

Table2. Coordinates of significant sources (MNI). The coordinates of the highest statistical values for each of the contrasts are shown. 
Source space analysis was performed over the significant time-windows from the sensor analysis. Paired-sample t-tests were computed between the conditions in order to investigate for significant differences between the conditions. In line with the results in sensor space, after selecting the time-windows already implicated by the sensor analysis, the cluster-based permutation test revealed a significant difference between the number strings and the pseudowords. These differences were most pronounced over the temporal lobe and the DLPFC (dorsolateral prefrontal cortex) of the left hemisphere. When testing for effects between number strings and false font strings, the cluster-based permutation test revealed significant difference which was most pronounced over the DLPFC and premotor of the left hemisphere and over the DLPFC, and inferior frontal gyrus of the right hemisphere. No significant effects were present in source space when testing for the greater response of false font strings, compared with number strings, in source space ( $p>0.05)$. When comparing pseudowords with false font strings, the clusterbased permutation test revealed a significant effect which was most pronounced over the fusiform, and the temporal lobe of the right hemisphere. No significant effects were evidenced at the source space when testing for higher power values of pseudowords, compared with false font strings, at the source space ( $p>0.05)$.

The results of Experiment 2 in the source space (Figure 5) showed medium and large effects. Means (M), standard deviations (SD), effect sizes and correlation values of the three conditions (numbers, letters, false-fonts), that result in the significant sources at the significant time-windows (tw) in the sensor space are depicted as follows: comparison 1) numbers $(\mathrm{M}=1.4 ; \mathrm{SD}=.22)$ versus letters $(\mathrm{M}=1.82 ; \mathrm{SD}=.44)$, tw: 264-500,effect size: 1.19, $\mathrm{r}=.38$; comparison 2) numbers $(\mathrm{M}=2.76 ; \mathrm{SD}=.57)$ versus false fonts $(\mathrm{M}=2.13$; $\mathrm{SD}=.41)$, tw: 93-191 ms, effect size: $-1.24, \mathrm{r}=.54$; comparison 3) letters $(\mathrm{M}=2.81$; $\mathrm{SD}=.68)$ versus false fonts $(\mathrm{M}=3.97 ; \mathrm{SD}=1 . .43)$, tw: $171-262 \mathrm{~ms}$, effect size: $1.03, \mathrm{r}=.59$.

\section{Discussion}

In the current study, we investigated the neurophysiological response to visually presented numbers in healthy young adults. Our data show that single numbers elicit an early ( $<200 \mathrm{~ms})$ preferential recruitment of neuronal populations over occipito-temporal regions bilaterally (Grotheer et al., 2016). Furthermore, while strings of numbers 
dissociate preferentially from pseudowords at later time-windows (with the higher differences shown over the left temporal regions, $>260 \mathrm{~ms}$ ) they dissociate from falsefont strings in earlier time windows $(<200 \mathrm{~ms}$, with the higher differences over the prefrontal and temporal sensors bilaterally). The approach used in the current study, which combines high temporal resolution, a whole-head coverage of the surface of the brain and a source localization of the effects, offers further data about how numbers dissociate from letters and false-fonts. Together, the present data contribute to the notion that the adult human visual system dissociates between these culturally-created symbols at the earliest encoding levels (Park et al., 2015).

Bilateral early preference of numbers. The triple-code-hypothesis (Dehaene, 1995, 1997) postulates that numbers are encoded as strings of digits on an internal visuospatial scratchpad which involve bilateral OT regions of the ventral visual pathway. In support for this, recent evidence shows a preferential response to numbers (compared to physically similar stimuli) over these regions (Park, Hebrank, et al., 2012; Roux et al., 2008). In their iEEG study, Shum et al., (2013) revealed a highly selective response to numerals in the right inferior temporal gyrus, anterior to the occipital temporal incisures. Although most of the electrodes in their patients were implanted in the right hemisphere, they were able to see a similar pattern in the left hemisphere as well. More recently, Groetheer et al., (2016) localized a preferential BOLD response for numbers at the inferior temporal gyrus. Interestingly, this pattern was present in both hemispheres.

This evidence agrees with our current results showing a bilateral preference for single numbers when comparing with letters and false-fonts (Experiment 1). A possible explanation for the bilateral preference during number processing originates from the 'biased connectivity' hypothesis. Under this hypothesis, category-specific visual areas emerge at cortical sites that exhibit a higher density of white-matter fiber tracts to and from the cortical circuits that are crucial for the target task. In the case of the number form area (NFA) primary target circuits would be the bilateral intraparietal sites that encode non-symbolic numerical quantities (Hannagan et al., 2015). This hypothesis is based on previous data that links connectivity patterns to functional specialization in the symbol form areas. A recent study by Abboud et al., (2015) reveals high connectivity patterns between the NFA and regions involved in representing quantities such as the intraparietal sulcus (IPS). The IPS is involved in the supramodal representation of numbers and is activated bilaterally more strongly when processing numbers than when processing letters 
(Eger et al., 2003) or false-fonts (Woodhead et al., 2011). Together, the bilateral occipitotemporal preference during number processing could represent a low-level visual processing which drives (via structural and/or functional connectivity) the preferred activation to the higher order areas.

Importantly, the effects in Experiment 1 (single stimuli) showed a peak at early latencies of the visual encoding (between $124 \mathrm{~ms}$ and $210 \mathrm{~ms}$ ). This time-window could be explained by the so-called $\mathrm{N} 1$ event-related potential component which is related with the visual encoding and discrimination of visual categories (Rossion, Joyce, Cottrell, \& Tarr, 2003; Tanaka, Luu, Weisbrod, \& Kiefer, 1999). Supporting our results, Dehaene (1996) showed that participants elicited more bilateral N1 activity when engaged in a numerical than in a verbal task.

Together with our results, the most pronounced discrimination (or perceptual specialization) of single numbers, letters forms and false-fonts took place over occipitotemporal regions at the earliest stages of the visual encoding level.

Our data also showed that the dissociation between number strings and false-fonts took place bilaterally early in the visual encoding (93-191 ms) (Experiment 2). Interestingly, the dissociation was more pronounced over the PFC bilaterally (temporal, parietal and occipital sensors were also highlighted). The PFC is involved in semantic association, as shown by Diester et and Niedel (2007). In their study, they trained monkeys to assign visual shapes to numerical categories and recorded from single cells in the prefrontal and parietal regions of the brain. The resulting data showed that the learned numerical value of the visual shapes was encoded by the neurons in the PFC. Furthermore, the data allowed them to propose this region as a neuronal precursor for number symbol encoding. Consistent with this evidence, the greater recruitment of bilateral PFC regions for number-strings may represent the semantic nature of these culturally-learned symbols in comparison with the meaningless symbols. In addition, similarly to previous literature on word processing (Wheat et al., 2010; Woodhead et al., 2012), the current findings could suggest top-down effects from the inferior frontal gyrus to the ventral occipito-temporal cortex during number-string processing.

Contrary to the bilateral preference for numbers, previous literature has shown a right lateralization of the brain activity when processing these symbols. In an EEG investigation, Park et al., (2014) reported a double dissociation when comparing single 
stimuli. While numbers activated right sensors more than letters, letters activated left sensors more than numbers. The discrepancy between the studies could be caused by the different methodological approaches used in the analyses. While we used a cluster-based permutation test taking all the sensors into account, Park and collaborators restricted their analysis to two preselected temporal-occipital channels. However, similar to our data, the topography of the raw data in their study evidences a bilateral pattern for numbers. In a similar manner, Carreiras et al. (2015) observed higher ERP responses to number strings over right occipito-temporal regions when comparing with consonant strings. In addition to the different methodological approaches used, the nature of the stimuli could also explain the discrepancies between studies. In a fMRI study, Abboud et al. (2015) used a complex numerosity task and showed a preferential activation for number identification in the right inferior temporal gyrus. However, the uncorrected results also revealed activation of the homologous left gyrus which suggested a possible role of this hemisphere on the identification of numerosity. As mentioned before, the neural response to numbers has been difficult to identify with fMRI due to a high rate of signal loss in this area. To compensate for that, the authors excluded the voxels with the lowest signal strength. This procedure may have hindered bilateral effects and contribute to the discrepancies between studies. Future studies which combine high temporal and spatial resolution techniques together with whole-brain methodological approaches will help to clarify these discrepancies.

Left hemispheric preference for letters and pseudowords. According to previous literature (Dehaene, 1995; Polk et al., 2002; Reinke et al., 2008; Vartiainen et al., 2011; Price, 2012), our data (Experiment 1) show left lateralized preference for single letters over the left inferior PFC during the first encoding levels (130-210 ms), when comparing with single false fonts.

As part of the language-processing network, the left inferior prefrontal gyrus is implicated, through a top-down processing to the ventral occipito-temporal cortex, in the integration of general visual form recognition, especially in the processing of visual word forms, (Cai et al., 2010; Wheat et al., 2010; Woodhead et al., 2012). Supported by the connectivity biased between the left temporal cortex and the left inferior frontal gyrus hypothesis (mentioned earlier), the current results could indicate preferential feedback mechanisms between these two regions when processing letter forms. Further studies which include connectivity measures will evaluate such a hypothesis. 
As far as we are aware of, the preferential response of the inferior PFC to letters or pseudowords (in comparison with false-fonts and numbers, respectively) is a novel result. One of the main reasons of its novelty could be that previous investigations focused their analyses only on the posterior regions of the brain. For example, Park et al., (2012) restricted their analysis to parietal, occipital and temporal regions of interest (ROI) and include only strings of stimuli. Park et al., (2014) restricted their analysis to the posterior sensors which showed the largest effects, as described before. Groetheer et al., (2016) established regions of interest on the right and left number form areas (covering mainly the inferior temporal gyrus), and the iEEG study by Shum et al., (2013) was restricted to the location of the intracranial electrodes (which covered mostly right temporal and only some left temporal regions). However, similar to our results, Carreiras et al., (2015) did observe higher ERF amplitudes in the inferior PFC when processing pseudowords than when processing consonant strings, suggesting a higher activation of this region for higher-pronounceable characters than for less-pronounceable characters. Interestingly, the Z-scores shown in their results suggest a potentially preferential neural response for pseudowords over the left hemisphere when comparing with strings of numbers (as shown in our study).

In the Experiment 2, pseudowords elicited a left lateralized preference when compared with number strings. Interestingly, this dissociation emerged in later time-windows (264$500 \mathrm{~ms}$ ) and showed the largest effect over the left temporal cortex, including the fusiform gyrus, and the left inferior PFC. The left inferior temporal cortex is shown to be sensitive to visual word forms (McCandliss et al., 2003). Furthermore, the preferential recruitment of the left fusiform and temporal gyri during word form processing is a robust finding in the literature (Cohen et al., 2002; Binder et al., 2006; Baker et al., 2007;) and is consistent across orthographies (Dehaene and Cohen, 2011). Park et al., (2012, 2014) have recently investigated with fMRI and EEG how the brain dissociates between letters and numbers. Similar to our results, their participants also recruited the left fusiform and inferior temporal gyri more when processing letters than when processing numbers.

Importantly, the dissociation between pseudowords and number strings in our study was more prominent starting approximately $264 \mathrm{~ms}$. This time-window is coincident with the so-called P2 event-related potential component. This ERP component is a positive deflection starting approximately $250 \mathrm{~ms}$ after stimulus onset and is modulated by the 
linguistic aspects of the stimuli, such as phonology and semantics (Barber et al., 2004, Carreiras et al.,2005; Hauk et al., 2006). During lexical decision tasks, pseudowords elicit larger amplitude responses than words in the P2 component (Hauk et al., 2006). During semantic tasks, words elicit larger amplitudes than consonant strings (McCandliss et al., 1997). Based on this, our results showing a difference in the amplitude between 264-500 ms between pseudowords and number strings but not between single letters and numbers could suggest that the visual cortex may be implicitly extracting phonological or semantic information when processing word-like stimuli. Park et al., (2014) obtained a similar pattern of results and elegantly suggested that such results could be explained by a later stage of a hierarchy of local combination detectors (Dehaene et al., 2005). Under this hypothesis, combinations of characters (such as pseudowords) may be processed more effectively by neurons in the higher levels of the visual-word-form processing, while neurons in the lower levels may process single characters. Such a hypothesis should be addressed in future investigations.

Right occipito-temporal preference for false-fonts. It is noteworthy to mention that falsefont strings preferentially recruited right occipito-temporal regions between 171-262 ms when comparing with pseudowords (Experiment 2). At a first glance, these results might seem somewhat unexpected. However, enhanced activity to non-nameable stimuli such as pseudowords has been previously observed (Park et al., 2014; Park, Hebrank, et al., 2012; Vinckier et al., 2007) over regions of the right hemisphere (Beason-Held et al., 1998; Haxby et al., 1995).

As mentioned earlier, the occipito-temporal region is a brain area sensitive to visual word forms. It is an area where categories, like words, are first identified and where the linguistic aspects of the stimuli commence after to be extracted. This pattern of activity seems to suggest that unfamiliar objects require more processing for identification and categorization (Appelbaum et al., 2009; Herdman, 2011; Herdman and Takai, 2013). Potentially, the localization and latency of the effects could potentially show an inefficient (and implicit) extraction of phonological or semantic information from the unknown stimuli. On a similar view and consistent with the study by Park, et al. (2012) in which they observed a larger fMRI response to false fonts than to letters in a study of monozygotic twins, the current results could be explained by an inefficiency in the template-matching process for unfamiliar stimuli that propagates through later phases of the processing. 
Conclusion. The results obtained in the current study suggest that the visual cortex (and importantly also prefrontal regions) discriminates between numbers, letters and falsefonts at early stages of the visual encoding. Our data show the importance of combining high temporal and spatial resolution techniques in order to fully understand the mechanisms underlying such dissociation. Together with previous evidence, our data point towards a new example of acquired category-specific responses in the human visual system. Future investigations will evaluate the current results and will contribute to the knowledge on how experience tunes the visual system for category recognition.

\section{Data availability statement}

The data generated during the current study are publicly archived at the following URL: https://openneuro.org/datasets/ds001985/versions/1.0.0

\section{Acknowledgments}

The research was partially supported by Basque Government (BERC 2018-2021 program), BCBL Severo Ochoa excellence accreditation SEV-2015-0490, and Grant RTI2018-093547-B-I00 from the Agencia Estatal de Investigación.

\section{References}

Abboud S., Maidenbaum S., Dehaene S., Amedi A. (2015). A number-form area in the blind. Nat. Commun, 6:6026.

Anderson SW, Damasio AR, Damasio H. (1990). Troubled letters but not numbersDomain specific cognitive impairments following focal damage in frontal-cortex. Brain, 113:749-766.

Appelbaum, L. G., Liotti, M., Perez, R., III, Fox, S. P., \& Woldorff, M. G. (2009). The temporal dynamics of implicit processing of non-letter, letter, and word-forms in the human visual cortex. Frontiers in Human Neuroscience, 3, 56.

L.L. Beason,Held, K.P. Purpura, J.W. Van,Meter, N.P. Azari, D.J. Mangot, L.M.Optica n, M.J. Mentis, G.E. Alexander, C.L. Grady, B. Horwitz, et al. (1998). PET reveals 
occipitotemporal pathway activation during elementary form perception in humans. Vis. Neurosci, 15, 503-510

Benjamin, D. J., Berger, J. O., Johannesson, M., Nosek, B. A., Wagenmakers, E. J., Berk, R., ... \& Cesarini, D. (2018). Redefine statistical significance. Nature Human Behaviour, 2(1), 6 .

Button, K. S., Ioannidis, J. P., Mokrysz, C., Nosek, B. A., Flint, J., Robinson, E. S., \& Munafò, M. R. (2013). Power failure: why small sample size undermines the reliability of neuroscience. Nature Reviews Neuroscience, 14(5), 365.

Carreiras M, Monahan PJ, Lizarazu M, Duñabeitia JA, Molinaro N. (2015). Numbers are not like words: Different pathways for literacy and numeracy. Neuroimage, Sep, 118:79-89

Cohen, L., Lehericy, S., Chochon, F., Lemer, C., Rivaud, S., Dehaene, S. (2002). Language-specific tuning of visual cortex functional properties of the Visual Word Form Area. Brain 125, 1054-1069

Baker, C.I., Liu, J., Wald, L.L., Kwong, K.K., Benner, T., Kanwisher, N. (2007). Visual word processing and experiential origins of functional selectivity in human extrastriate cortex. Proc. Natl. Acad. Sci. U. S. A. 104, 9087-9092.

Barber, H.A., Vergara, M., \& Carreiras, M., (2004) Syllable-frequency effects in visual word recognition: Evidence from ERPs. Neuroreport, 15(3), 545-548.

Binder, J.R., Medler, D.A., Westbury, C.F., Liebenthal, E., Buchanan, L. (2006). Tuning of the human left fusiform gyrus to sublexical orthographic structure. NeuroImage 33, 739-748

Cai, Q., Paulignan, Y., Brysbaert, M., Ibarrola, D., Nazir, T.A. (2010). The left ventral occipitotemporal response to words depends on language lateralization but not on visual familiarity. Cereb. Cortex 20, 1153-1163.

Carreiras, M., Vergara, M., \& Barber, H., (2005) Early ERP effects of syllabic processing during visual word recognition. Journal of Cognitive Neuroscience, 17(11), 18031817

Carreiras M, Monahan PJ, Lizarazu M, Duñabeitia JA, Molinaro N. (2015). Numbers are not like words: Different pathways for literacy and numeracy. Neuroimage. 118:79-89

Chein JM, Ravizza SM, Fiez J. (2003). Using neuroimaging to eval- uate models of working memory and their implications for language processing. $\mathrm{J}$ Neurolinguistics 16:315-339. 
Cohen, L., \& Dehaene, S. (1995). Number processing in pure alexia: the effect of hemispheric asymmetries and task demands. NeuroCase, 1, 121-137.

Cohen, L., \& Dehaene, S. (1996). Cerebral networks for number processing: Evidence from a case of posterior callosal lesion. NeuroCase, 2, 155-174.

Dehaene, S. (1996). The organization of brain activations in number comparison: Eventrelated potentials and the additive-factors methods. Journal of Cognitive Neuroscience, 8, 47-68.

Dehaene, S. (1997). The number sense. New York: Oxford University Press.

Dehaene S, Cohen L. (2007). Cultural recycling of cortical maps. Neuron, 56:384-398.

Diester I, Nieder A (2007) Semantic associations between signs and numerical categories in the prefrontal cortex. PLoS Biol 5(11):e294.

Eger E, Sterzer P, Russ MO, Giraud AL, Kleinschmidt A. (2003). A supramodal number representation in human intraparietal cortex. Neuron, 37:719-725.

Fiez JA, Balota DA, Raichle ME, Petersen SE. (1999). Effects of lexicality, frequency, and spelling-to-sound consistency on the functional anatomy of reading. Neuron 24:205-218.

Gelman, A., \& Carlin, J. (2014). Beyond power calculations: assessing type S (sign) and type M (magnitude) errors. Perspectives on Psychological Science, 9(6), 641-651.

Greenland, S., Senn, S. J., Rothman, K. J., Carlin, J. B., Poole, C., Goodman, S. N., \& Altman, D. G. (2016). Statistical tests, P values, confidence intervals, and power: a guide to misinterpretations. European journal of epidemiology, 31(4), 337-350.

Grotheer M, Herrmann KH, Kovács G (2016) Neuroimaging evidence of a bilateral representation for visually presented numbers. J Neurosci 36:88-97

Hamilton JP, Mirkin M, Polk TA. (2006). Category-level contributions to the alphanumeric category effect in visual search. Psychonomic Bulletin \& Review, 13:1074-1077.

Hannagan T., Amedi A., Cohen L., Dehaene-Lambertz G., Dehaene S. (2015). Origins of the specialization for letters and numbers in ventral occipitotemporal cortex. Trends Cogn. Sci, 19(7):374-382.

Hauk O, Davis MH, Ford M, Pulvermuller F, Marslen-Wilson WD. (2006). The time course of visual word recognition as revealed by linear regression analysis of ERP data. NeuroImage, 30(4):1383-1400.

Haxby JV, Ungerleider LG, Horwitz B, Rapoport SI, (1995).GradyHemispheric differences in neural systems for face working memory: A PET-rCBF Study 
Human Brain Mapp., 3, 68-82

James KH, James TW, Jobard G, Wong AC, Gauthier I. (2005). Letter processing in the visual system: different activation patterns for single letters and strings. Cognitive, Affective \& Behavioral Neuroscience. 5(4):452-66.

Lakens, D., Adolfi, F. G., Albers, C. J., Anvari, F., Apps, M. A., Argamon, S. E., ... \& Buchanan, E. M. (2018). Justify your alpha. Nature Human Behaviour, 2(3), 168.

Maris, E., \& Oostenveld, R. (2007). Nonparametric statistical testing of EEG-and MEGdata. Journal of neuroscience methods, 164(1), 177-190.

McCandliss BD, Posner MI, Givon T. (1997). Brain plasticity in learning visual words. Cognitive Psychology, 33:88-110.

McCandliss BD, Cohen L, Dehaene S. (2003). The visual word form area: Expertise for reading in the fusiform gyrus. Trends in Cognitive Sciences, 7:293-299.

Oostenveld, R., Fries, P., Maris, E., Schoffelen, JM (2011) FieldTrip: Open Source Software for Advanced Analysis of MEG, EEG, and Invasive Electrophysiological Data. Computational Intelligence and Neuroscience Volume

Park J, Hebrank A, Polk TA, Park DC. (2012). Neural dissociation of number from letter recognition and its relationship to parietal numerical processing. Journal of Cognitive Neuroscience, 24(1):39-50.

Park J, Chiang C, Brannon EM, Woldorff MG (2014) Experience-dependent hemispheric specialization of letters and numbers is revealed in early visual processing. J Cogn Neurosci 26:2239-2249

Polk, T.A., Stallcup, M., Aguirre, G.K., Alsop, D.C., D'Esposito, M., Detre, J.A., Farah, M.J. (2002). Neural specialization for letter recognition. J. Cogn. Neurosci. 14, $145-159$

Price, C.J. (2012). A review and synthesis of the first 20 years of PET and fMRI studies of heard speech, spoken language and reading. NeuroImage 62, 816-847.

Reinke, K., Fernandes, M., Schwindt, G., O'Craven, K., Grady, C.L. (2008). Functional specificity of the visual word form area: general activation for words and symbols but specific network activation for words. Brain Lang. 104, 180-189.

Rossion B, Joyce CA, Cottrell GW, Tarr MJ. (2003). Early lateralization and orientation tuning for face, word, and object processing in the visual cortex. Neuroimage, Nov;20(3):1609-24. 
Roux FE, Lubrano V, Lauwers-Cances V, Giussani C, Démonet JF. (2008). Cortical areas involved in Arabic number reading. Neurology, 70:210-217

Schendan, H. E., Ganis, G., \& Kutas, M. (1998). Neurophysiological evidence for visual perceptual categorization of words and faces within $150 \mathrm{msec}$. Psychophysiology, $35,240-251$.

Schlaggar BL, McCandliss BD. (2007). Development of neural systems for reading. Annual Review of Neuroscience, 30:475-503.

Seymour, S. E., Reuter-Lorenz, P. A., \& Gazzaniga, M. S. (1994). The disconnection syndrome: basic findings reaffirmed. Brain, 117, 105-115.

Shum J, Hermes D, Foster BL, Dastjerdi M, Rangarajan V, Winawer J, Miller KJ, Parvi zi J (2013) A brain area for visual numerals. J Neurosci 33:6709-6715

Smith EE, Jonides J. (1999). Storage and executive processes in the frontal lobes. Science 283:1657-1661

Tanaka JW, Luu P, Weisbrod M, Kiefer M. (1999). Tracking the time course of object categorization using event-related potentials. NeuroReport, 10:829-835

Taulu S and Simola J. (2006). Spatiotemporal signal space separation method for rejecting nearby interference in MEG measurements. Phys Med Biol 51, 17591768.

Taulu S. and Kajola K. (2005). Presentation of electromagnetic multichannel data: The signal space separation method. Journal of applied physics, 97, 124905

Vartiainen, J., Liljestrom, M., Koskinen, M., Renvall, H., Salmelin, R. (2011). Functional magnetic resonance imaging blood oxygenation level-dependent signal and magnetoencephalography evoked responses yield different neural functionality in reading. J. Neurosci. 31, 1048-1058

Vinckier F, Dehaene S, Jobert A, Dubus JP, Sigman M, Cohen L. (2007). Hierarchical coding of letter strings in the ventral stream: dissecting the inner organization of the visual word-form system. Neuron, 55(1):143-156

Wasserstein, R. L., \& Lazar, N. A. (2016). The ASA's statement on p-values: context, process, and purpose.

Wheat, K.L., Cornelissen, P.L., Frost, S.J., Hansen, P.C. (2010). During visual word recognition, phonology is accessed within $100 \mathrm{~ms}$ and may be mediated by a speech production code: evidence from magnetoencephalography. J. Neurosci. $30,5229-5233$ 
Woodhead, Z.V., Barnes, G.R., Penny, W., Moran, R., Teki, S., Price, C.J., Leff, A.P., (2012). Reading front to back: MEG evidence for early feedback effects during word recognition. Cereb. Cortex 24, 817-825.

Zhang, Jian and Liu, Chao (2015) Linearly Constrained Minimum Variance Beamforming. Journal of Machine Learning Research. ISSN 1532-4435.

Zoe V.J. Woodhead, Sonia L. E. Brownsett, Novraj S. Dhanjal, Christian Beckmann, Richard J. S. Wise (2011). The Visual Word Form System in Context. Journal of Neuroscience, 31 (1) 193-199 


\section{Acknowledgments}

The research was partially supported by Basque Government (BERC 2018-2021 program), BCBL Severo Ochoa excellence accreditation SEV-2015-0490, and Grant RTI2018-093547-B-I00 from the Agencia Estatal de Investigación. 